BULLETIN OF THE

AMERICAN MATHEMATICAL SOCIETY

Volume 78, Number 4, July 1972

\title{
NONASSOCIATIVE ADDITION OF UNBOUNDED OPERATORS AND A PROBLEM OF BREZIS AND PAZY
}

\author{
BY PAUL R. CHERNOFF 1
}

Communicated by M. H. Protter, November 20, 1971

\begin{abstract}
We give a negative solution to a problem raised by Brezis and Pazy in the theory of nonlinear semigroups by relating it to a nonassociative phenomenon in the theory of addition of unbounded operators.
\end{abstract}

In their study of nonlinear contraction semigroups, Brezis and Pazy $[1$, p. 260] state the following problem:

"Let $A, B$ and $A+B$ be maximal monotone sets and $F(t), G(t) \in$ $\operatorname{Cont}(C)$ such that

$$
\begin{aligned}
& \lim _{t \rightarrow 0}(I+(\lambda / t)(I-F(t)))^{-1} x=(I+\lambda A)^{-1} x, \\
& \lim _{t \rightarrow 0}(I+(\lambda / t)(I-G(t)))^{-1} x=(I+\lambda B)^{-1} x
\end{aligned}
$$

for every $\lambda>0, x \in C$. Does

$$
\lim _{t \rightarrow 0}(I+(\lambda / t)(I-F(t) G(t)))^{-1} x=(I+\lambda(A+B))^{-1} x
$$

hold for every $\lambda>0, x \in C$ ?"

Here $C$ is a closed convex set in a Hilbert space and Cont $(C)$ is the set of nonexpansive mappings of $C$ into itself. Monotone sets are related to the (possibly multi-valued) generators of nonlinear contraction semigroups; however, in this note we will work only with linear semigroups, so we omit the detailed definition.

The answer to the above question is no, even in the linear theory, and even if $B$ is assumed to be 0 . It is quite interesting that this negative result is due to the failure of the associative law in generalized addition of operators; for this see [3].

To make the connection with product formulas, we note that (1) is equivalent to

$$
\lim _{n \rightarrow \infty} F(t / n)^{n} x=e^{t A} x
$$

for all $x$, uniformly on compact $t$ intervals. For present purposes we require this only for linear operators; $e^{t A}$ is the $\left(C_{0}\right)$ contraction semigroup generated by $A$. This result is discussed in [2] and [4, Theorem IX, 3.6]; a nonlinear version is [1, Theorem 3.4]. In [2] it is shown that (4) holds if $A$ is the closure of the strong derivative $F^{\prime}(0)$, generalizing the original theorem of Trotter [5].

AMS 1970 subject classifications. Primary 47B25, 47H99; Secondary 47D05.

1 Partially supported by National Science Foundation grant GP-25082.

Copyright (C) American Mathematical Society 1972 
We are now ready for the counterexample. Pick any skew-symmetric operator $S$ on Hilbert space which admits two distinct skew-adjoint extensions $A$ and $A^{\prime}$; such operators are well known to exist in profusion. Take $F(t)=e^{t A}$, the unitary group generated by $A$. Take $G(t)=e^{-t A} e^{t A^{\prime}}$. Note that the closure of $A^{\prime}-A$ is 0 . Hence by Trotter's theorem

$$
G(t / n)^{n} \rightarrow I
$$

in the strong operator topology, uniformly on compact $t$ intervals; thus

(2) holds with $B=0$. It is quite trivial that

$$
\begin{aligned}
F(t / n)^{n} & =\left(e^{t / n A}\right)^{n} \rightarrow e^{t A}, \\
(F(t / n) G(t / n))^{n} & =\left(e^{t / n A} e^{-t / n A} e^{t / n A^{\prime}}\right)^{n} \rightarrow e^{t A^{\prime}} .
\end{aligned}
$$

In other words (1) holds but (3) fails. In fact the analogue of (3) holds with $A^{\prime}$ replacing $A+B=A$.

As we pointed out in [3] this example illustrates the failure of the associative law for addition followed by closing:

$$
A=\left[A+\left(-A+A^{\prime}\right)^{-}\right] \neq\left[(A-A)^{-}+A^{\prime}\right]=A^{\prime} .
$$

\section{REFERENCES}

1. H. Brezis and A. Pazy, Semigroups of nonlinear contractions on convex sets, J. Functional Analysis 6 (1970), 237-281.

2. P. R. Chernoff, Note on product formulas for operator semigroups, J. Functional Analysis 2 (1968), 238-242. MR 37 \# 6793.

3. - Semigroup product formulas and addition of unbounded operators, Bull. Amer. Math. Soc. 76 (1970), 395-398. MR 41 \# 2457.

4. T. Kato, Perturbation theory for linear operators, Die Grundlehren der math. Wissenschaften, Band 132, Springer-Verlag, New York, 1966. MR 34 \#3324.

5. H. F. Trotter, On the product of semi-groups of operators, Proc. Amer. Math. Soc. 10 (1959), 545-551. MR 21 \# 7446.

Department of Mathematics, University of California, Berkeley, California 94720 